\title{
Coding function for non-coding RNA in plants-insights from miRNA encoded peptide (miPEP)
}

\author{
WANG Long $^{1} \&$ WANG Jia-Wei ${ }^{1,2^{*}}$ \\ ${ }^{1}$ National Key Laboratory of Plant Molecular Genetics, Institute of Plant Physiology and Ecology, Shanghai Institutes for Biological Sciences, \\ Shanghai 200032, China; \\ ${ }^{2}$ ShanghaiTech University, Shanghai 200031, China
}

Received April 2, 2015; accepted April 9, 2015; published online April 21, 2015

Citation: Wang L, Wang JW. Coding function for non-coding RNA in plants-insights from miRNA encoded peptide (miPEP). Sci China Life Sci, 2015, 58: 503-505, doi: 10.1007/s11427-015-4854-z

Non-coding RNAs (ncRNAs) refer to a large and diverse collection of polyadenylated or non-polyadenylated RNA transcripts with low protein coding potential. In general, the house-keeping ncRNAs (e.g., ribosomal RNAs and transfer RNAs), primary transcripts of microRNAs (miRNAs) and plant specific RNA polymerase $\mathrm{V}$ (Pol V)-dependent ncRNA transcripts also fall into this definition. The application of next-generation sequencing leads to identification of over 30,000 putative ncRNAs in plants [1]. While it is possible that some ncRNAs are transcription noises or the by-products of RNA processing and degradation, growing lines of evidence suggest that a large fraction of them are functional and play various regulatory activities during development.

Recent studies have revealed the role of long ncRNAs (lncRNAs) in regulating gene expression. IPSI (INDUCED BY PHOSPHATE STARVATIONI) encodes a ncRNA with a short motif that is highly complementary to the sequence of miR399 except a mismatched loop at the expected miR399 cleavage site. The mismatch loop enables IPS1 to function as a miRNA target mimicry (non-degradable miRNA target) and sequestrate miR399 activity [2]. Pol V-dependent ncRNA transcripts have been implicated in RNA-directed DNA methylation, which indirectly causes gene silencing [3]. IncRNA can also regulate gene expression by recruiting

*Corresponding author (email: jwwang@ @ibs.ac.cn) histone modification complex. FLOWERING LOCUS C (FLC), a MADS-box transcription factor, negatively regulates flowering in Arabidopsis thaliana. In response to cold, the expression of FLC is greatly reduced largely due to the epigenetic silencing mediated by $\mathrm{H} 3 \mathrm{~K} 27$ trimethylation. Dean and Sung labs identified two cold-responsive IncRNAs, COLDAIR and COOLAIR, transcribed by independent promoters from the FLC genomic region [4]. While the COOLAIR transcript is physically associated with FLC locus and accelerates transcriptional shutdown of FLC during cold exposure, COLDAIR directly interacts with the Polycomb repressive complex 2 to induce epigenetic repression of FLC. More recently, two papers from Crespi lab demonstrated that lncRNA can hijack nuclear alternative splicing regulators to modulate alternative splicing patterns [5] or regulate the formation of a chromatin loop encompassing the promoter of its neighboring gene [6].

Surprisingly, a new study now demonstrated that the primary transcript of miRNA has potential coding function. Lauressergues and his colleagues [7] identified miPEPs, the short peptides encoded by pri-miRNAs in Arabidopsis and Medicago truncatula (Figure 1). Immunoblot using the specific antibodies validated that at least five predicted miPEPs are naturally translated in plants.

Promoter reporter analyses revealed that the miPEPs confer the same expression pattern as their associated mature miRNAs. miPEPs are not junks or by-products, rather 


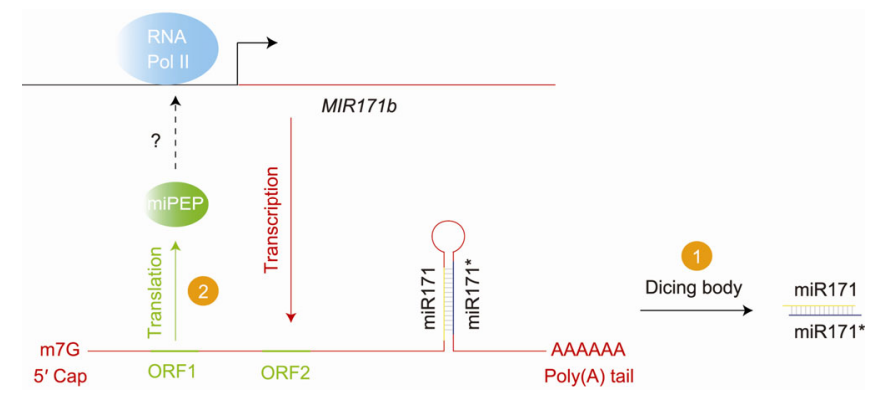

Figure 1 (color online) Biogenesis of miRNA and miPEP. MIRI71B is used as an example [7]. The pri-miR171b, transcribed by RNA Pol II, undergoes fate decisions: It can be processed by dicing body to produce miRNA duplex or translated into miPEP (ORF1) which in turn increases the transcription of MIRI71B. The dashed line indicates unknown molecular mechanism.

they have specific biological function. Lauressergues et al. found that overexpression of miPEP171b in M. truncatula leads to the increased accumulation of endogenous miR171b, which in turn modifies root development (Figure 1). This result is further confirmed by application of synthetic miPEP171b to M. truncatula. Notably, the activation of miRNA by corresponding miPEP is a general phenomenon. Application of the synthetic peptide or overexpression of five additional miPEPs from different miRNA families in M. truncatula and A. thaliana increases accumulation of the corresponding miRNAs.

The increased amount of mature miRNAs caused by miPEP overexpression can result from either enhanced pri-miRNA procession or increased miRNA transcription. Treatment with cordycepin, an inhibitor of RNA synthesis, completely abolishes the positive effect of miPEP on pri-miRNA accumulation, suggesting that miPEP increases miRNA abundance through activating the transcription of miRNAs.

The discovery of miPEPs is in agreement with the recent genome-wide studies showing hundreds of micropeptides encoded in vertebrate lncRNAs. Although Lauressergues et al. provided compelling evidence that miPEPs exert potential role in regulating miRNA expression, several fundamental questions need to be resolved. First, how does miPEP function? In Arabidopsis, there is no common signature among miPEPs, suggesting each of these putative miPEPs is likely specific for its miRNA. These findings raise the question about how miPEP regulates transcription of pri-miRNA. Does miPEP function as a complex with other unknown factors? Whether they regulate miRNA expression in trans or in cis?

As messenger RNAs (mRNAs) for protein coding genes, pri-miRNAs are transcribed by RNA polymerase II and harbor 5'7-methylguanosine cap and polyadenylate tail [8]. The identification of miPEP suggests that pri-miRNA is subjected to a fate decision between translation and miRNA procession. Indeed, genome-wide profiling of ribosome footprints in Arabidopsis found that the sequence encoding miPEP165a in pri-miR165a is occupied by ribosomes [9]. However, it remains unclear that how the translation of miPEP is coordinated with miRNA procession and why a fraction of pri-miRNAs is not processed by Dicing body in nucleus.

Bioinformatic analyses predict that most pri-miRNAs are able to encode more than one miPEP. Lauressergues et al. found that there are the two putative open reading frames (ORFs) of pri-miR171b in M. truncatula and only the first ORF can be translated in to a small peptide. Do all miPEPs function as transcriptional activators? A possible strategy for determining their functions is to overexpress candidate miPEPs and monitor changes in gene expression and phenotypes. While being more convincing, the reverse experiment by removal of endogenous coding sequence for miPEP is challenging because it is difficult to determine whether any changes in gene expression or phenotypes are due to loss of the coding sequence or disruption of the transcript in which it lies.

Another unanswered question is, given their small size, whether miPEPs can move between cells or be transported in a long-distance manner. In Arabidopsis, it is prevalent that small secreted peptides mediate short or long-distance signaling, as exemplified by CLAVATA3 peptides in the maintenance of shoot apical meristem and CEP peptides in coordinating root development with needs for nitrogen [10]. Thus, the comparison of the localization of pri-mRNAs and related miPEPs will elucidate whether miPEP acts in a cell-autonomous or non-cell-autonomous way.

As always, the unexpected is the most fascinating. Further experimental validation of miPEPs in other plants, identification of the molecular mechanism by which miPEP regulates gene expression, and delineation of miPEP evolution will be important next steps.

1 Liu J, Wang H, Chua NH. Long noncoding RNA transcriptome of plants. Plant Biotechnol J, 2015, doi: 10.1111/pbi.12336

2 Franco-Zorrilla JM, Valli A, Todesco M, Mateos I, Puga MI, Rubio-Somoza I, Leyva A, Weigel D, Garcia JA, Paz-Ares J. Target mimicry provides a new mechanism for regulation of microRNA activity. Nat Genet, 2007, 39: 1033-1037

3 Matzke MA, Kanno T, Matzke AJ. RNA-Directed DNA methylation: the evolution of a complex epigenetic pathway in flowering plants. Annu Rev Plant Biol, 2014, 10.1146/annurev-arplant-043014-114633

4 Song J, Irwin J, Dean C. Remembering the prolonged cold of winter. Curr Biol, 2013, 23: R807-811

5 Bardou F, Ariel F, Simpson CG, Romero-Barrios N, Laporte P, Balzergue S, Brown JW, Crespi M. Long noncoding RNA modulates alternative splicing regulators in Arabidopsis. Dev Cell, 2014, 30: $166-176$

6 Ariel F, Jegu T, Latrasse D, Romero-Barrios N, Christ A, Benhamed M, Crespi M. Noncoding transcription by alternative RNA polymerases dynamically regulates an auxin-driven chromatin loop. Mol Cell, 2014, 55: 383-396

7 Lauressergues D, Couzigou JM, Clemente HS, Martinez Y, Dunand C, Becard G, Combier JP. Primary transcripts of microRNAs encode 
regulatory peptides. Nature, 2015, doi: 10.1038/nature14346

8 Rogers $\mathrm{K}$, Chen X. Biogenesis, Turnover, and mode of action of plant microRNAs. Plant Cell, 2013, 25: 2383-2399

9 Juntawong P, Girke T, Bazin J, Bailey-Serres J. Translational dynamics revealed by genome-wide profiling of ribosome footprints in Arabidopsis. Proc Natl Acad Sci USA, 2014, 111: E203-212

10 Tabata R, Sumida K, Yoshii T, Ohyama K, Shinohara H, Matsubayashi Y. Perception of root-derived peptides by shoot LRRRKs mediates systemic N-demand signaling. Science, 2014, 346: 343-346

Open Access This article is distributed under the terms of the Creative Commons Attribution License which permits any use, distribution, and reproduction in any medium, provided the original author(s) and source are credited. 